\title{
Chebyshev Distance
}

\author{
Roland Coghetto \\ Rue de la Brasserie 5 \\ 7100 La Louvière, Belgium
}

\begin{abstract}
Summary. In 21, Marco Riccardi formalized that $\mathbb{R N}$-basis $n$ is a basis (in the algebraic sense defined in [26]) of $\mathcal{E}_{T}^{n}$ and in [20] he has formalized that $\mathcal{E}_{T}^{n}$ is second-countable, we build (in the topological sense defined in 23]) a denumerable base of $\mathcal{E}_{T}^{n}$.

Then we introduce the $n$-dimensional intervals (interval in $n$-dimensional Euclidean space, pavé (borné) de $\mathbb{R}^{n}\left[16\right.$, semi-intervalle (borné) de $\mathbb{R}^{n}[22$ ).

We conclude with the definition of Chebyshev distance [11.
\end{abstract}

MSC: 54E35 03B35

Keywords: second-countable; intervals; Chebyshev distance

MML identifier: SRINGS_5, version: 8.1.04 5.36.1267

\section{Preliminaries}

From now on $n$ denotes a natural number, $r, s$ denote real numbers, $x, y$ denote elements of $\mathcal{R}^{n}, p, q$ denote points of $\mathcal{E}_{\mathrm{T}}^{n}$, and $e$ denotes a point of $\mathcal{E}^{n}$.

Now we state the propositions:

(1) $|x-y|=|y-x|$.

(2) Let us consider a natural number $i$. If $i \in \operatorname{Seg} n$, then $|x|(i) \in \mathbb{R}$.

(3) Let us consider elements $x, y$ of $\mathbb{R}$, and extended reals $x_{1}, y_{1}$. If $x \leqslant x_{1}$ and $y \leqslant y_{1}$, then $x+y \leqslant x_{1}+y_{1}$.

(4) Let us consider real numbers $a, c$, and an extended real number $b$. Suppose $a<b$ and $[a, b[\subseteq[a, c[$. Then $b$ is a real number.

(5) Let us consider a non empty set $D$, and a non empty subset $D_{1}$ of $D$. Then $D_{1}^{n} \subseteq D^{n}$. 
(6) Let us consider a non empty set $X$, and a function $f$. Suppose $f=$ $\operatorname{Seg} n \longmapsto X$. Then $f$ is a non-empty, $n$-element finite sequence.

Let $n$ be a natural number. The functor $\mathbb{R}(n)$ yielding a non-empty, $n$ element finite sequence is defined by the term

(Def. 1) $\operatorname{Seg} n \longmapsto \mathbb{R}$.

Now we state the propositions:

(7) $\mathbb{R}(n)=\operatorname{Seg} n \longmapsto$ the carrier of $\mathbb{R}^{\mathbf{1}}$.

(8) $\prod(\operatorname{Seg} n \longmapsto \mathbb{R})=\mathcal{R}^{n}$.

(9) $\prod \mathbb{R}(n)=\mathcal{R}^{n}$.

(10) Let us consider a set $X$. Then $\prod(\operatorname{Seg} n \longmapsto X)=X^{n}$.

(11) Let us consider a non empty set $D$, and an $n$-tuple $x$ of $D$. Then $x \in$ $D^{\operatorname{Seg} n}$.

(12) Let us consider a subset $O_{1}$ of $\mathcal{E}_{\mathrm{T}}^{n}$, and a subset $O_{2}$ of $\left(\mathcal{E}^{n}\right)_{\text {top }}$. If $O_{1}=O_{2}$, then $O_{1}$ is open iff $O_{2}$ is open.

(13) Suppose $e=p$. Then the set of all OpenHypercube $\left(e, \frac{1}{m}\right)$ where $m$ is a non zero element of $\mathbb{N}=$ the set of all OpenHypercube $\left(p, \frac{1}{m}\right)$ where $m$ is a non zero element of $\mathbb{N}$.

(14) If $q \in$ OpenHypercube $(p, r)$, then $p \in \operatorname{OpenHypercube}(q, r)$.

(15) If $q \in$ OpenHypercube $\left(p, \frac{r}{2}\right)$, then OpenHypercube $\left(q, \frac{r}{2}\right) \subseteq$ OpenHypercube $(p, r)$.

Let $x$ be an element of $\mathbb{R} \times \mathbb{R}$. The functors: $(x)_{1}$ and $(x)_{2}$ yield elements of $\mathbb{R}$. Let $n$ be a natural number and $x$ be an element of $\mathcal{R}^{n} \times \mathcal{R}^{n}$. The functors: $(x)_{1}$ and $(x)_{2}$ yield elements of $\mathcal{R}^{n}$. Now we state the proposition:

(16) Let us consider an $n$-element finite sequence $f$ of elements of $\mathbb{R} \times \mathbb{R}$. Then there exists an element $x$ of $\mathcal{R}^{n} \times \mathcal{R}^{n}$ such that for every natural number $i$ such that $i \in \operatorname{Seg} n$ holds $(x)_{\mathbf{1}}(i)=\left(f_{i}\right)_{\mathbf{1}}$ and $(x)_{\mathbf{2}}(i)=\left(f_{i}\right)_{\mathbf{2}}$.

\section{The Set of $n$-Tuples of Rational Numbers}

Let us consider $n$. The functor $\mathcal{Q}^{n}$ yielding a set of finite sequences of $\mathbb{Q}$ is defined by the term

(Def. 2) $\mathbb{Q}^{n}$.

Now we state the proposition:

(17) $\mathcal{Q}^{0}=\{0\}$.

One can check that $\mathcal{Q}^{0}$ is trivial.

Let us consider $n$. One can check that $\mathcal{Q}^{n}$ is non empty and every element of $\mathcal{Q}^{n}$ is $n$-element and $\mathcal{Q}^{n}$ is countable. 
Let $n$ be a positive natural number. Let us note that $\mathcal{Q}^{n}$ is infinite and $\mathcal{Q}^{n}$ is denumerable.

Now we state the proposition:

(18) $\mathcal{Q}^{n}$ is a dense subset of $\mathcal{E}_{\mathrm{T}}^{n}$.

ProOF: $\mathcal{Q}^{n}$ is a subset of $\mathcal{R}^{n}$. Reconsider $R=\mathcal{Q}^{n}$ as a subset of $\mathcal{E}_{\mathrm{T}}^{n}$. For every subset $Q$ of $\mathcal{E}_{\mathrm{T}}^{n}$ such that $Q \neq \emptyset$ and $Q$ is open holds $R$ meets $Q$ by [10, (67)], (12), [15, (23)], [13, (39)].

Let us consider $n$. One can check that $\mathcal{Q}^{n}$ is countable and dense as a subset of $\mathcal{E}_{\mathrm{T}}^{n}$.

\section{A Countable Base of an $n$-Dimensional Euclidean Space}

(VERSION 1: [20]):

Let $n$ be a natural number. Let us observe that there exists a basis of $\mathcal{E}_{\mathrm{T}}^{n}$ which is countable.

Let us consider $n$ and $e$. Note that OpenHypercubes $e$ is countable.

The functor OpenHypercubes- $\mathbb{Q}(n)$ yielding a non empty set is defined by the term

(Def. 3) $\left\{\right.$ OpenHypercubes $q$, where $q$ is a point of $\left.\mathcal{E}^{n}: q \in \mathcal{Q}^{n}\right\}$.

Let $q$ be an element of $\mathcal{Q}^{n}$. The functor ${ }^{\circledR} q$ yielding a point of $\mathcal{E}^{n}$ is defined by the term

(Def. 4) $q$.

Let $q$ be an object. Assume $q \in \mathcal{Q}^{n}$. The functor object $2 \mathbb{Q}(q, n)$ yielding an element of $\mathcal{Q}^{n}$ is defined by the term

(Def. 5) $q$.

Let us note that OpenHypercubes- $\mathbb{Q}(n)$ is countable and $\cup$ OpenHypercubes- $\mathbb{Q}(n)$ is countable.

Now we state the propositions:

(19) UOpenHypercubes- $\mathbb{Q}(n)$ is an open family of subsets of $\mathcal{E}_{\mathrm{T}}^{n}$. The theorem is a consequence of (12).

(20) Let us consider a non empty, open subset $O$ of $\mathcal{E}_{\mathrm{T}}^{n}$. Then there exists an element $p$ of $\mathcal{Q}^{n}$ such that $p \in O$. The theorem is a consequence of (18).

(21) Let us consider a family $\mathcal{B}$ of subsets of $\mathcal{E}_{\mathrm{T}}^{n}$.

Suppose $\mathcal{B}=\bigcup$ OpenHypercubes- $\mathbb{Q}(n)$. Then $\mathcal{B}$ is quasi basis.

Proof: $F$ is quasi basis by (12), [15, (23)], [10, (67)], (20).

Let us consider $n$. Observe that $\bigcup$ OpenHypercubes- $\mathbb{Q}(n)$ is non empty. 
The functor OpenHypercubes $\mathbb{Q} U$ nion $(n)$ yielding a countable, open family of subsets of $\mathcal{E}_{\mathrm{T}}^{n}$ is defined by the term

(Def. 6) U OpenHypercubes- $\mathbb{Q}(n)$.

Now we state the proposition:

(22) OpenHypercubes $\mathbb{Q} U$ nion $(n)=\left\{\right.$ OpenHypercube $\left(q, \frac{1}{m}\right)$,

where $q$ is a point of $\mathcal{E}^{n}, m$ is a positive natural number : $\left.q \in \mathcal{Q}^{n}\right\}$.

(VERSION 2):

Let $n$ be a natural number. Observe that there exists a basis of $\mathcal{E}_{\mathrm{T}}^{n}$ which is countable.

Now we state the propositions:

(23) OpenHypercubes $\mathbb{Q} U$ nion $(n)$ is a countable basis of $\mathcal{E}_{\mathrm{T}}^{n}$.

(24) Let us consider an open subset $O$ of $\mathcal{E}_{\mathrm{T}}^{n}$. Then there exists a subset $Y$ of OpenHypercubes $\mathbb{Q} U$ nion $(n)$ such that

(i) $Y$ is countable, and

(ii) $O=\bigcup Y$.

The theorem is a consequence of (21).

Let us consider an open, non empty subset $O$ of $\mathcal{E}_{\mathrm{T}}^{n}$. Now we state the propositions:

(25) There exists a subset $Y$ of OpenHypercubes $\mathbb{Q} U n i o n(n)$ such that

(i) $Y$ is not empty, and

(ii) $O=\bigcup Y$, and

(iii) there exists a function $g$ from $\mathbb{N}$ into $Y$ such that for every object $x$, $x \in O$ iff there exists an object $y$ such that $y \in \mathbb{N}$ and $x \in g(y)$.

The theorem is a consequence of (24).

(26) There exists a sequence $s$ of OpenHypercubes $\mathbb{Q} U n i o n(n)$ such that for every object $x, x \in O$ iff there exists an object $y$ such that $y \in \mathbb{N}$ and $x \in s(y)$. The theorem is a consequence of (25).

(27) There exists a sequence $s$ of OpenHypercubes $\mathbb{Q} U n i o n(n)$ such that $O=$ $\cup s$. The theorem is a consequence of (26).

\section{The Set of All Left Open Real Bounded Intervals}

The set of all left open real bounded intervals yielding a family of subsets of $\mathbb{R}$ is defined by the term

(Def. 7) the set of all $] a, b]$ where $a, b$ are real numbers. 
Let us note that the set of all left open real bounded intervals is non empty. Now we state the propositions:

(28) The set of all left open real bounded intervals $\subseteq\{I$, where $I$ is a subset of $\mathbb{R}: I$ is left open interval\}.

(29) The set of all left open real bounded intervals is $\cap$-closed and $\backslash f p^{-c l o s e d}$ and has the empty element and countable cover.

(30) The set of all left open real bounded intervals is a semiring of $\mathbb{R}$.

\section{The Set of All Right Open Real Bounded Intervals}

The set of all right open real bounded intervals yielding a family of subsets of $\mathbb{R}$ is defined by the term

(Def. 8) the set of all $[a, b[$ where $a, b$ are real numbers.

Observe that the set of all right open real bounded intervals is non empty.

Now we state the propositions:

(31) The set of all right open real bounded intervals $\subseteq\{I$, where $I$ is a subset of $\mathbb{R}: I$ is right open interval\}.

(32) The set of all right open real bounded intervals has the empty element.

(33) (i) the set of all right open real bounded intervals is $\cap$-closed, and

(ii) the set of all right open real bounded intervals is $\backslash f p^{-c l o s e d}$ and has the empty element.

The theorem is a consequence of (31), (32), and (4).

(34) The set of all right open real bounded intervals has countable cover.

Proof: Define $\mathcal{F}$ [object, object] $\equiv \$_{1}$ is an element of $\mathbb{N}$ and $\$_{2} \in$ the set of all right open real bounded intervals and there exists a real number $x$ such that $x=\$_{1}$ and $\$_{2}=[-x, x[$. For every object $x$ such that $x \in \mathbb{N}$ there exists an object $y$ such that $y \in$ the set of all right open real bounded intervals and $\mathcal{F}[x, y]$. Consider $f$ being a function such that $\operatorname{dom} f=\mathbb{N}$ and rng $f \subseteq$ the set of all right open real bounded intervals and for every object $x$ such that $x \in \mathbb{N}$ holds $\mathcal{F}[x, f(x)]$ from [7, Sch. 6]. rng $f$ is countable by [27, (4)], [14, (58)]. $\operatorname{rng} f$ is a cover of $\mathbb{R}$ by [2, (2)], [12, (8)], [3, (13)], [17, (45)].

(35) The set of all right open real bounded intervals is a semiring of $\mathbb{R}$. 


\section{Finite Product of Left Open Intervals}

In the sequel $n$ denotes a non zero natural number.

Let $n$ be a non zero natural number. The functor LeftOpenIntervals $(n)$ yielding a classical semiring family of $\mathbb{R}(n)$ is defined by the term

(Def. 9) $\operatorname{Seg} n \longmapsto$ (the set of all left open real bounded intervals).

Now we state the propositions:

(36) LeftOpenIntervals $(n)=\operatorname{Seg} n \longmapsto$ the set of all $] a, b]$ where $a, b$ are real numbers.

(37) MeasurableRectangle LeftOpenIntervals $(n)$ is a semiring of $\mathcal{R}^{n}$. The theorem is a consequence of (8).

Let us consider an object $x$.

Let us assume that $x \in$ MeasurableRectangle LeftOpenIntervals $(n)$. Now we state the propositions:

(38) There exists a subset $y$ of $\mathcal{R}^{n}$ such that

(i) $x=y$, and

(ii) for every natural number $i$ such that $i \in \operatorname{Seg} n$ there exist real numbers $a, b$ such that for every element $t$ of $\mathcal{R}^{n}$ such that $t \in y$ holds $t(i) \in] a, b]$.

The theorem is a consequence of (37).

(39) There exists a subset $y$ of $\mathcal{R}^{n}$ and there exists an $n$-element finite sequence $f$ of elements of $\mathbb{R} \times \mathbb{R}$ such that $x=y$ and for every element $t$ of $\mathcal{R}^{n}, t \in y$ iff for every natural number $i$ such that $i \in \operatorname{Seg} n$ holds $\left.t(i) \in]\left(f_{i}\right)_{\mathbf{1}},\left(f_{i}\right)_{\mathbf{2}}\right]$.

Proof: MeasurableRectangle LeftOpenIntervals $(n)$ is a family of subsets of $\mathcal{R}^{n}$. Reconsider $y=x$ as a subset of $\mathcal{R}^{n}$. Consider $g$ being a function such that $x=\prod g$ and $g \in \prod$ LeftOpenIntervals $(n)$. Define $\mathcal{P}$ [natural number, set $] \equiv$ there exists an element $x$ of $\mathbb{R} \times \mathbb{R}$ such that $\$_{2}=x$ and $\left.\left.g\left(\$_{1}\right)=\right](x)_{\mathbf{1}},(x)_{\mathbf{2}}\right]$. For every natural number $i$ such that $i \in \operatorname{Seg} n$ there exists an element $d$ of $\mathbb{R} \times \mathbb{R}$ such that $\mathcal{P}[i, d]$. There exists a finite sequence $f_{1}$ of elements of $\mathbb{R} \times \mathbb{R}$ such that len $f_{1}=n$ and for every natural number $i$ such that $i \in \operatorname{Seg} n$ holds $\mathcal{P}\left[i, f_{1_{i}}\right]$ from [25, Sch. 1]. Consider $f_{1}$ being a finite sequence of elements of $\mathbb{R} \times \mathbb{R}$ such that len $f_{1}=n$ and for every natural number $i$ such that $i \in \operatorname{Seg} n$ there exists an element $x$ of $\mathbb{R} \times$ $\mathbb{R}$ such that $f_{1 i}=x$ and $\left.\left.g(i)=\right](x)_{\mathbf{1}},(x)_{\mathbf{2}}\right]$. For every natural number $i$ such that $i \in \operatorname{Seg} n$ holds $\left.g(i)=]\left(f_{1 i}\right)_{\mathbf{1}},\left(f_{1 i}\right)_{\mathbf{2}}\right]$. For every element $t$ of $\mathcal{R}^{n}$ such that $t \in y$ for every natural number $i$ such that $i \in \operatorname{Seg} n$ holds $\left.t(i) \in]\left(f_{1 i}\right)_{\mathbf{1}},\left(f_{1 i}\right)_{\mathbf{2}}\right]$. For every element $t$ of $\mathcal{R}^{n}$ such that for every natural 
number $i$ such that $i \in \operatorname{Seg} n$ holds $\left.t(i) \in]\left(f_{1_{i}}\right)_{\mathbf{1}},\left(f_{1_{i}}\right)_{\mathbf{2}}\right]$ holds $t \in y$ by $[6$, $(93)]$.

(40) There exists a subset $y$ of $\mathcal{R}^{n}$ and there exist elements $a, b$ of $\mathcal{R}^{n}$ such that $x=y$ and for every object $s, s \in y$ iff there exists an element $t$ of $\mathcal{R}^{n}$ such that $s=t$ and for every natural number $i$ such that $i \in \operatorname{Seg} n$ holds $t(i) \in] a(i), b(i)]$. The theorem is a consequence of (39) and (16).

Now we state the proposition:

(41) Let us consider a set $x$. Suppose $x \in$ MeasurableRectangle LeftOpenInter$\operatorname{vals}(n)$. Then there exist elements $a, b$ of $\mathcal{R}^{n}$ such that for every element $t$ of $\mathcal{R}^{n}, t \in x$ iff for every natural number $i$ such that $i \in \operatorname{Seg} n$ holds $t(i) \in] a(i), b(i)]$. The theorem is a consequence of (39) and (16).

\section{Finite Product of Right Open Intervals}

Let $n$ be a non zero natural number. The functor RightOpenIntervals $(n)$ yielding a classical semiring family of $\mathbb{R}(n)$ is defined by the term

(Def. 10) Seg $n \longmapsto$ (the set of all right open real bounded intervals).

From now on $n$ denotes a non zero natural number.

Now we state the propositions:

(42) RightOpenIntervals $(n)=\operatorname{Seg} n \longmapsto$ the set of all $[a, b$ [ where $a, b$ are real numbers.

(43) MeasurableRectangle RightOpenIntervals $(n)$ is a semiring of $\mathcal{R}^{n}$. The theorem is a consequence of (8).

Let us consider an object $x$.

Let us assume that $x \in$ MeasurableRectangle RightOpenIntervals $(n)$. Now we state the propositions:

(44) There exists a subset $y$ of $\mathcal{R}^{n}$ such that

(i) $x=y$, and

(ii) for every natural number $i$ such that $i \in \operatorname{Seg} n$ there exist real numbers $a, b$ such that for every element $t$ of $\mathcal{R}^{n}$ such that $t \in y$ holds $t(i) \in[a, b[$.

The theorem is a consequence of (43).

(45) There exists a subset $y$ of $\mathcal{R}^{n}$ and there exists an $n$-element finite sequence $f$ of elements of $\mathbb{R} \times \mathbb{R}$ such that $x=y$ and for every element $t$ of $\mathcal{R}^{n}, t \in y$ iff for every natural number $i$ such that $i \in \operatorname{Seg} n$ holds $t(i) \in\left[\left(f_{i}\right)_{\mathbf{1}},\left(f_{i}\right)_{\mathbf{2}}[\right.$.

Proof: MeasurableRectangle RightOpenIntervals $(n)$ is a family of subsets of $\mathcal{R}^{n}$. Reconsider $y=x$ as a subset of $\mathcal{R}^{n}$. Consider $g$ being a function 
such that $x=\prod g$ and $g \in \prod$ RightOpenIntervals $(n)$. Define $\mathcal{P}$ [natural number, set $] \equiv$ there exists an element $x$ of $\mathbb{R} \times \mathbb{R}$ such that $\$_{2}=x$ and $g\left(\$_{1}\right)=\left[(x)_{\mathbf{1}},(x)_{\mathbf{2}}[\right.$. For every natural number $i$ such that $i \in \operatorname{Seg} n$ there exists an element $d$ of $\mathbb{R} \times \mathbb{R}$ such that $\mathcal{P}[i, d]$. There exists a finite sequence $f_{1}$ of elements of $\mathbb{R} \times \mathbb{R}$ such that len $f_{1}=n$ and for every natural number $i$ such that $i \in \operatorname{Seg} n$ holds $\mathcal{P}\left[i, f_{1_{i}}\right]$ from [25, Sch. 1]. Consider $f_{1}$ being a finite sequence of elements of $\mathbb{R} \times \mathbb{R}$ such that len $f_{1}=n$ and for every natural number $i$ such that $i \in \operatorname{Seg} n$ there exists an element $x$ of $\mathbb{R} \times$ $\mathbb{R}$ such that $f_{1 i}=x$ and $g(i)=\left[(x)_{\mathbf{1}},(x)_{\mathbf{2}}[\right.$. For every natural number $i$ such that $i \in \operatorname{Seg} n$ holds $g(i)=\left[\left(f_{1 i}\right)_{\mathbf{1}},\left(f_{1 i}\right)_{\mathbf{2}}[\right.$. For every element $t$ of $\mathcal{R}^{n}$ such that $t \in y$ for every natural number $i$ such that $i \in \operatorname{Seg} n$ holds $t(i) \in\left[\left(f_{1 i}\right)_{\mathbf{1}},\left(f_{1 i}\right)_{\mathbf{2}}\left[\right.\right.$. For every element $t$ of $\mathcal{R}^{n}$ such that for every natural number $i$ such that $i \in \operatorname{Seg} n$ holds $t(i) \in\left[\left(f_{1_{i}}\right)_{\mathbf{1}},\left(f_{1_{i}}\right)_{\mathbf{2}}\right.$ [ holds $t \in y$ by [6, (93)].

(46) There exists a subset $y$ of $\mathcal{R}^{n}$ and there exist elements $a, b$ of $\mathcal{R}^{n}$ such that $x=y$ and for every object $s, s \in y$ iff there exists an element $t$ of $\mathcal{R}^{n}$ such that $s=t$ and for every natural number $i$ such that $i \in \operatorname{Seg} n$ holds $t(i) \in[a(i), b(i)[$. The theorem is a consequence of (45) and (16).

Now we state the proposition:

(47) Let us consider a set $x$. Suppose $x \in$ MeasurableRectangle RightOpenIntervals $(n)$. Then there exist elements $a, b$ of $\mathcal{R}^{n}$ such that for every element $t$ of $\mathcal{R}^{n}, t \in x$ iff for every natural number $i$ such that $i \in \operatorname{Seg} n$ holds $t(i) \in[a(i), b(i)[$. The theorem is a consequence of (45) and (16).

\section{8. $n$-Dimensional Product of Subset Family}

In the sequel $n$ denotes a natural number, $X$ denotes a set, and $S$ denotes a family of subsets of $X$.

Let us consider $n$ and $X$. The functor $\operatorname{Product}(n, X)$ yielding a set is defined by

(Def. 11) for every object $f, f \in i$ iff there exists a function $g$ such that $f=\prod g$ and $g \in \prod(\operatorname{Seg} n \longmapsto X)$.

Now we state the propositions:

(48) $\operatorname{Product}(n, X) \subseteq 2^{(\bigcup \bigcup(\operatorname{Seg} n \longmapsto X))^{\operatorname{dom}(\operatorname{Seg} n \longmapsto X)}}$.

(49) $\operatorname{Product}(n, S)$ is a family of subsets of $\prod(\operatorname{Seg} n \longmapsto X)$.

Proof: Reconsider $S_{1}=\operatorname{Product}(n, S)$ as a subset of $2^{(\bigcup \bigcup(\operatorname{Seg} n \longmapsto S))^{\operatorname{dom}(\operatorname{Seg} n \longmapsto S)} . S_{1} \subseteq{ }_{2} \prod(\operatorname{Seg} n \longmapsto X)}$ by [1, (9)], [24, (13), (7)], [9. (77), (81)]. 
(50) Let us consider a non empty family $S$ of subsets of $X$. Then $\operatorname{Product}(n, S)=$ the set of all $\prod f$ where $f$ is an $n$-tuple of $S$.

Proof: $\operatorname{Product}(n, S) \subseteq$ the set of all $\prod f$ where $f$ is an $n$-tuple of $S$ by (10), [6, (131)]. the set of all $\prod f$ where $f$ is an $n$-tuple of $S \subseteq \operatorname{Product}(n, S)$ by [6, (131)], (10).

(51) Let us consider a non zero natural number $n$. Then $\operatorname{Product}(n, X) \subseteq$ $2^{(\bigcup X)^{\operatorname{Seg} n}}$.

Let us consider a non zero natural number $n$, a non empty set $X$, and a non empty family $S$ of subsets of $X$.

Let us assume that $S \neq\{\emptyset\}$. Now we state the propositions:

(52) $\operatorname{Product}(n, S) \subseteq 2^{X^{\operatorname{Seg} n}}$. The theorem is a consequence of (51) and (5).

(53) UProduct $(n, S) \subseteq X^{\operatorname{Seg} n}$. The theorem is a consequence of (52).

Let $n$ be a natural number and $X$ be a non empty set. Let us note that $\operatorname{Product}(n, X)$ is non empty.

Now we state the proposition:

(54) Let us consider a non empty set $X$, a non empty family $S$ of subsets of $X$, and a subset $x$ of $X^{\operatorname{Seg} n}$. Then $x$ is an element of $\operatorname{Product}(n, S)$ if and only if there exists an $n$-tuple $s$ of $S$ such that for every element $t$ of $X^{\operatorname{Seg} n}$, for every natural number $i$ such that $i \in \operatorname{Seg} n$ holds $t(i) \in s(i)$ iff $t \in x$.

\section{The Set of All Closed Real Bounded Intervals}

The set of all closed real bounded intervals yielding a family of subsets of $\mathbb{R}$ is defined by the term

(Def. 12) the set of all $[a, b]$ where $a, b$ are real numbers.

Now we state the proposition:

(55) The set of all closed real bounded intervals $=\{I$, where $I$ is a subset of $\mathbb{R}: I$ is closed interval $\}.$

Let us note that the set of all closed real bounded intervals is non empty.

Now we state the propositions:

(56) The set of all closed real bounded intervals is $\cap$-closed and has the empty element and countable cover.

Proof: The set of all closed real bounded intervals is $\cap$-closed. There exists a countable subset $X$ of the set of all closed real bounded intervals such that $X$ is a cover of $\mathbb{R}$ by [27, (4)], [14, (58)], [2, (2)], [12, (8)].

(57) Let us consider a natural number $n$. Then Seg $n \longmapsto$ (the set of all closed real bounded intervals) is an $n$-element finite sequence. 


\section{The Set of All Open Real Bounded Intervals}

The set of all open real bounded intervals yielding a family of subsets of $\mathbb{R}$ is defined by the term

(Def. 13) the set of all $] a, b[$ where $a, b$ are real numbers.

Now we state the proposition:

(58) The set of all open real bounded intervals $\subseteq\{I$, where $I$ is a subset of $\mathbb{R}: I$ is open interval $\}$.

Let us observe that the set of all open real bounded intervals is non empty. Now we state the propositions:

(59) The set of all open real bounded intervals is $\cap$-closed and has the empty element and countable cover.

Proof: The set of all open real bounded intervals is $\cap$-closed. There exists a countable subset $X$ of the set of all open real bounded intervals such that $X$ is a cover of $\mathbb{R}$ by [27, (4)], [14, (58)], [2, (2)], [12, (8)].

(60) Let us consider a natural number $n$. Then $\operatorname{Seg} n \longmapsto$ (the set of all open real bounded intervals) is an $n$-element finite sequence.

\section{1. $n$-Dimensional Subset Family of $\mathbb{R}$}

From now on $n$ denotes a natural number and $S$ denotes a family of subsets of $\mathbb{R}$.

Now we state the proposition:

(61) $\operatorname{Product}(n, S)$ is a family of subsets of $\mathcal{R}^{n}$. The theorem is a consequence of (49) and (8).

Let us consider $n$ and $S$. One can check that the functor Product $(n, S)$ yields a family of subsets of $\mathcal{R}^{n}$. Now we state the propositions:

(62) Let us consider a non empty family $S$ of subsets of $\mathbb{R}$, and a subset $x$ of $\mathcal{R}^{n}$. Then $x$ is an element of $\operatorname{Product}(n, S)$ if and only if there exists an $n$-tuple $s$ of $S$ such that for every element $t$ of $\mathcal{R}^{n}$, for every natural number $i$ such that $i \in \operatorname{Seg} n$ holds $t(i) \in s(i)$ iff $t \in x$.

Proof: If $x$ is an element of $\operatorname{Product}(n, S)$, then there exists an $n$-tuple $s$ of $S$ such that for every element $t$ of $\mathcal{R}^{n}$, for every natural number $i$ such that $i \in \operatorname{Seg} n$ holds $t(i) \in s(i)$ iff $t \in x$ by [․ (93)]. If there exists an $n$ tuple $s$ of $S$ such that for every element $t$ of $\mathcal{R}^{n}$, for every natural number $i$ such that $i \in \operatorname{Seg} n$ holds $t(i) \in s(i)$ iff $t \in x$, then $x$ is an element of $\operatorname{Product}(n, S)$ by [6, (93)]. 
(63) Let us consider a non zero natural number $n$, and an $n$-tuple $s$ of the set of all closed real bounded intervals. Then there exist elements $a, b$ of $\mathcal{R}^{n}$ such that for every natural number $i$ such that $i \in \operatorname{Seg} n$ holds $s(i)=$ $[a(i), b(i)]$.

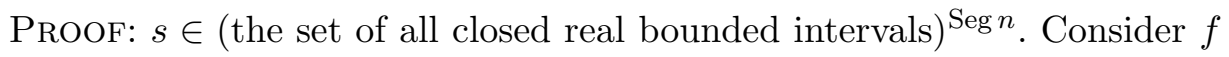
being a function such that $s=f$ and $\operatorname{dom} f=\operatorname{Seg} n$ and $\operatorname{rng} f \subseteq$ the set of all closed real bounded intervals. Define $\mathcal{P}$ [object, object] $\equiv$ there exists an element $f$ of $\mathbb{R} \times \mathbb{R}$ such that $f=\$_{2}$ and $s\left(\$_{1}\right)=\left[(f)_{\mathbf{1}},(f)_{\mathbf{2}}\right]$. For every natural number $i$ such that $i \in \operatorname{Seg} n$ there exists an element $d$ of $\mathbb{R} \times \mathbb{R}$ such that $\mathcal{P}[i, d]$ by [7, (3)]. Consider $f$ being a finite sequence of elements of $\mathbb{R} \times \mathbb{R}$ such that len $f=n$ and for every natural number $i$ such that $i \in \operatorname{Seg} n$ holds $\mathcal{P}\left[i, f_{i}\right]$ from [25, Sch. 1]. Consider $z$ being an element of $\mathcal{R}^{n} \times \mathcal{R}^{n}$ such that for every natural number $i$ such that $i \in \operatorname{Seg} n$ holds $(z)_{\mathbf{1}}(i)=\left(f_{i}\right)_{\mathbf{1}}$ and $(z)_{\mathbf{2}}(i)=\left(f_{i}\right)_{\mathbf{2}}$. Reconsider $a=(z)_{\mathbf{1}}, b=(z)_{\mathbf{2}}$ as an element of $\mathcal{R}^{n}$. For every natural number $i$ such that $i \in \operatorname{Seg} n$ holds $s(i)=[a(i), b(i)]$.

(64) Let us consider a non zero natural number $n$, and an element $x$ of Product ( $n$, the set of all closed real bounded intervals). Then there exist elements $a, b$ of $\mathcal{R}^{n}$ such that for every element $t$ of $\mathcal{R}^{n}, t \in x$ iff for every natural number $i$ such that $i \in \operatorname{Seg} n$ holds $t(i) \in[a(i), b(i)]$. The theorem is a consequence of (62) and (63).

Let us consider a non zero natural number $n$, a subset $x$ of $\mathcal{R}^{n}$, and elements $a, b$ of $\mathcal{R}^{n}$. Now we state the propositions:

(65) Suppose for every element $t$ of $\mathcal{R}^{n}, t \in x$ iff for every natural number $i$ such that $i \in \operatorname{Seg} n$ holds $t(i) \in[a(i), b(i)]$. Then $x$ is an element of Product ( $n$, the set of all closed real bounded intervals).

Proof: Define $\mathcal{P}$ [object, object] $\equiv$ there exists a natural number $n$ such that $\$_{1}=n$ and $\$_{2}=[a(n), b(n)]$. For every natural number $i$ such that $i \in \operatorname{Seg} n$ there exists an element $d$ of the set of all closed real bounded intervals such that $\mathcal{P}[i, d]$. There exists a finite sequence $g$ of elements of the set of all closed real bounded intervals such that len $g=n$ and for every natural number $i$ such that $i \in \operatorname{Seg} n$ holds $\mathcal{P}\left[i, g_{i}\right]$ from [25, Sch. 1]. Consider $g$ being a finite sequence of elements of the set of all closed real bounded intervals such that len $g=n$ and for every natural number $i$ such that $i \in \operatorname{Seg} n$ holds $\mathcal{P}\left[i, g_{i}\right]$. For every natural number $i$ such that $i \in \operatorname{Seg} n$ holds $g(i)=[a(i), b(i)]$. There exists a function $g$ such that $x=\prod g$ and $g \in \prod(\operatorname{Seg} n \longmapsto$ (the set of all closed real bounded intervals)) by [4, (89)], [24, (13), (7)], [1, (9)].

(66) Suppose for every element $t$ of $\mathcal{R}^{n}, t \in x$ iff for every natural number 
$i$ such that $i \in \operatorname{Seg} n$ holds $t(i) \in] a(i), b(i)]$. Then $x$ is an element of $\operatorname{Product}(n$, the set of all left open real bounded intervals).

Proof: Define $\mathcal{P}$ [object, object] $\equiv$ there exists a natural number $n$ such that $\$_{1}=n$ and $\left.\left.\$_{2}=\right] a(n), b(n)\right]$. For every natural number $i$ such that $i \in \operatorname{Seg} n$ there exists an element $d$ of the set of all left open real bounded intervals such that $\mathcal{P}[i, d]$. There exists a finite sequence $g$ of elements of the set of all left open real bounded intervals such that len $g=n$ and for every natural number $i$ such that $i \in \operatorname{Seg} n$ holds $\mathcal{P}\left[i, g_{i}\right]$ from [25, Sch. 1]. Consider $g$ being a finite sequence of elements of the set of all left open real bounded intervals such that len $g=n$ and for every natural number $i$ such that $i \in \operatorname{Seg} n$ holds $\mathcal{P}\left[i, g_{i}\right]$. For every natural number $i$ such that $i \in \operatorname{Seg} n$ holds $g(i)=] a(i), b(i)]$. There exists a function $g$ such that $x=\prod g$ and $g \in \prod(\operatorname{Seg} n \longmapsto$ (the set of all left open real bounded intervals)) by [4, (89)], [24, (13), (7)], [1, (9)].

(67) Suppose for every element $t$ of $\mathcal{R}^{n}, t \in x$ iff for every natural number $i$ such that $i \in \operatorname{Seg} n$ holds $t(i) \in[a(i), b(i)[$. Then $x$ is an element of $\operatorname{Product}(n$, the set of all right open real bounded intervals).

Proof: Define $\mathcal{P}$ [object, object] $\equiv$ there exists a natural number $n$ such that $\$_{1}=n$ and $\$_{2}=[a(n), b(n)[$. For every natural number $i$ such that $i \in \operatorname{Seg} n$ there exists an element $d$ of the set of all right open real bounded intervals such that $\mathcal{P}[i, d]$. There exists a finite sequence $g$ of elements of the set of all right open real bounded intervals such that len $g=n$ and for every natural number $i$ such that $i \in \operatorname{Seg} n$ holds $\mathcal{P}\left[i, g_{i}\right]$ from [25, Sch. 1]. Consider $g$ being a finite sequence of elements of the set of all right open real bounded intervals such that len $g=n$ and for every natural number $i$ such that $i \in \operatorname{Seg} n$ holds $\mathcal{P}\left[i, g_{i}\right]$. For every natural number $i$ such that $i \in \operatorname{Seg} n$ holds $g(i)=[a(i), b(i)[$. There exists a function $g$ such that $x=\prod g$ and $g \in \prod(\operatorname{Seg} n \longmapsto$ (the set of all right open real bounded intervals)) by [4, (89)], [24, (13), (7)], [1, (9)].

Now we state the propositions:

(68) Let us consider a non zero natural number $n$, and an $n$-tuple $s$ of the set of all left open real bounded intervals. Then there exist elements $a, b$ of $\mathcal{R}^{n}$ such that for every natural number $i$ such that $i \in \operatorname{Seg} n$ holds $s(i)=] a(i), b(i)]$.

Proof: $s \in$ (the set of all left open real bounded intervals) $^{\operatorname{Seg} n}$. Consider $f$ being a function such that $s=f$ and $\operatorname{dom} f=\operatorname{Seg} n$ and $\operatorname{rng} f \subseteq$ the set of all left open real bounded intervals. Define $\mathcal{P}$ [object, object] $\equiv$ there exists an element $f$ of $\mathbb{R} \times \mathbb{R}$ such that $f=\$_{2}$ and $\left.\left.s\left(\$_{1}\right)=\right](f)_{\mathbf{1}},(f)_{\mathbf{2}}\right]$. For every natural number $i$ such that $i \in \operatorname{Seg} n$ there exists an element $d$ of $\mathbb{R} \times \mathbb{R}$ such that $\mathcal{P}[i, d]$ by [7, (3)]. Consider $f$ being a finite sequence of 
elements of $\mathbb{R} \times \mathbb{R}$ such that len $f=n$ and for every natural number $i$ such that $i \in \operatorname{Seg} n$ holds $\mathcal{P}\left[i, f_{i}\right]$ from [25, Sch. 1]. Consider $z$ being an element of $\mathcal{R}^{n} \times \mathcal{R}^{n}$ such that for every natural number $i$ such that $i \in \operatorname{Seg} n$ holds $(z)_{\mathbf{1}}(i)=\left(f_{i}\right)_{\mathbf{1}}$ and $(z)_{\mathbf{2}}(i)=\left(f_{i}\right)_{\mathbf{2}}$. Reconsider $a=(z)_{\mathbf{1}}, b=(z)_{\mathbf{2}}$ as an element of $\mathcal{R}^{n}$. For every natural number $i$ such that $i \in \operatorname{Seg} n$ holds $s(i)=] a(i), b(i)]$.

(69) Let us consider a non zero natural number $n$, and an element $x$ of $\operatorname{Product}(n$, the set of all left open real bounded intervals). Then there exist elements $a, b$ of $\mathcal{R}^{n}$ such that for every element $t$ of $\mathcal{R}^{n}, t \in x$ iff for every natural number $i$ such that $i \in \operatorname{Seg} n$ holds $t(i) \in] a(i), b(i)]$. The theorem is a consequence of (62) and (68).

(70) Let us consider a non zero natural number $n$, and an $n$-tuple $s$ of the set of all right open real bounded intervals. Then there exist elements $a, b$ of $\mathcal{R}^{n}$ such that for every natural number $i$ such that $i \in \operatorname{Seg} n$ holds $s(i)=[a(i), b(i)[$.

Proof: $s \in$ (the set of all right open real bounded intervals) ${ }^{\operatorname{Seg} n}$. Consider $f$ being a function such that $s=f$ and $\operatorname{dom} f=\operatorname{Seg} n$ and $\operatorname{rng} f \subseteq$ the set of all right open real bounded intervals. Define $\mathcal{P}$ [object, object] $\equiv$ there exists an element $f$ of $\mathbb{R} \times \mathbb{R}$ such that $f=\$_{2}$ and $s\left(\$_{1}\right)=\left[(f)_{\mathbf{1}},(f)_{\mathbf{2}}[\right.$. For every natural number $i$ such that $i \in \operatorname{Seg} n$ there exists an element $d$ of $\mathbb{R} \times \mathbb{R}$ such that $\mathcal{P}[i, d]$ by [7, (3)]. Consider $f$ being a finite sequence of elements of $\mathbb{R} \times \mathbb{R}$ such that len $f=n$ and for every natural number $i$ such that $i \in \operatorname{Seg} n$ holds $\mathcal{P}\left[i, f_{i}\right]$ from [25, Sch. 1]. Consider $z$ being an element of $\mathcal{R}^{n} \times \mathcal{R}^{n}$ such that for every natural number $i$ such that $i \in \operatorname{Seg} n$ holds $(z)_{\mathbf{1}}(i)=\left(f_{i}\right)_{\mathbf{1}}$ and $(z)_{\mathbf{2}}(i)=\left(f_{i}\right)_{\mathbf{2}}$. Reconsider $a=(z)_{\mathbf{1}}, b=(z)_{\mathbf{2}}$ as an element of $\mathcal{R}^{n}$. For every natural number $i$ such that $i \in \operatorname{Seg} n$ holds $s(i)=[a(i), b(i)[$.

(71) Let us consider a non zero natural number $n$, and an element $x$ of Product( $n$, the set of all right open real bounded intervals). Then there exist elements $a, b$ of $\mathcal{R}^{n}$ such that for every element $t$ of $\mathcal{R}^{n}, t \in x$ iff for every natural number $i$ such that $i \in \operatorname{Seg} n$ holds $t(i) \in[a(i), b(i)[$. The theorem is a consequence of (62) and (70).

\section{Closed/Open/Left-Open/Right-Open - Hyper Interval}

From now on $n$ denotes a natural number and $a, b, c, d$ denote elements of $\mathcal{R}^{n}$.

Let us consider $n, a$, and $b$. The functor ClosedHyperInterval $(a, b)$ yielding a subset of $\mathcal{R}^{n}$ is defined by 
(Def. 14) for every object $x, x \in i t$ iff there exists an element $y$ of $\mathcal{R}^{n}$ such that $x=y$ and for every natural number $i$ such that $i \in \operatorname{Seg} n$ holds $y(i) \in$ $[a(i), b(i)]$.

The functor OpenHyperInterval $(a, b)$ yielding a subset of $\mathcal{R}^{n}$ is defined by

(Def. 15) for every object $x, x \in i t$ iff there exists an element $y$ of $\mathcal{R}^{n}$ such that $x=y$ and for every natural number $i$ such that $i \in \operatorname{Seg} n$ holds $y(i) \in$ ]$a(i), b(i)[$.

The functor LeftOpenHyperInterval $(a, b)$ yielding a subset of $\mathcal{R}^{n}$ is defined by

(Def. 16) for every object $x, x \in$ it iff there exists an element $y$ of $\mathcal{R}^{n}$ such that $x=y$ and for every natural number $i$ such that $i \in \operatorname{Seg} n$ holds $y(i) \in$ ]$a(i), b(i)]$.

The functor RightOpenHyperInterval $(a, b)$ yielding a subset of $\mathcal{R}^{n}$ is defined by

(Def. 17) for every object $x, x \in$ it iff there exists an element $y$ of $\mathcal{R}^{n}$ such that $x=y$ and for every natural number $i$ such that $i \in \operatorname{Seg} n$ holds $y(i) \in$ $[a(i), b(i)[$.

Now we state the proposition:

(72) ClosedHyperInterval $(a, a)=\{a\}$.

Proof: ClosedHyperInterval $(a, a) \subseteq\{a\}$ by [6, (124)].

$\{a\} \subseteq$ ClosedHyperInterval $(a, a)$.

Let us consider $n$ and $a$. Let us observe that ClosedHyperInterval $(a, a)$ is trivial.

Now we state the proposition:

(73) (i) OpenHyperInterval $(a, b) \subseteq \operatorname{LeftOpenHyperInterval}(a, b)$, and

(ii) OpenHyperInterval $(a, b) \subseteq \operatorname{RightOpenHyperInterval}(a, b)$, and

(iii) LeftOpenHyperInterval $(a, b) \subseteq \operatorname{ClosedHyperInterval}(a, b)$, and

(iv) RightOpenHyperInterval $(a, b) \subseteq$ ClosedHyperInterval $(a, b)$.

Let us consider $n, a$, and $b$. We say that $a \leqslant b$ if and only if

(Def. 18) for every natural number $i$ such that $i \in \operatorname{Seg} n$ holds $a(i) \leqslant b(i)$.

One can verify that the predicate is reflexive.

Now we state the propositions:

(74) If $a \leqslant b \leqslant c$, then $a \leqslant c$.

(75) If $a \leqslant c$ and $d \leqslant b$,

then ClosedHyperInterval $(c, d) \subseteq$ ClosedHyperInterval $(a, b)$.

(76) If $a \leqslant b$, then ClosedHyperInterval $(a, b)$ is not empty. The theorem is a consequence of (75) and (72). 
Let us consider $n, a$, and $b$. We say that $a<b$ if and only if

(Def. 19) for every natural number $i$ such that $i \in \operatorname{Seg} n$ holds $a(i)<b(i)$.

Now we state the propositions:

(77) If $a<b<c$, then $a<c$.

(78) If $b<a$ and $n$ is not zero, then ClosedHyperInterval $(a, b)$ is empty.

(79) $n \mapsto r$ is an element of $\mathcal{R}^{n}$.

Proof: Set $f=n \mapsto r . f \in \mathbb{R}^{\operatorname{Seg} n}$ by [6, (112), (93)].

Let us consider $n$ and $r$. Note that the functor $n \mapsto r$ yields an element of $\mathcal{R}^{n}$. One can check that the functor $\langle r\rangle$ yields an element of $\mathcal{R}^{1}$. Now we state the propositions:

(80) Let us consider a non zero natural number $n$, and a point $e$ of $\mathcal{E}^{n}$. Then there exists an element $a$ of $\mathcal{R}^{n}$ such that

(i) $a=e$, and

(ii) OpenHypercube $(e, r)=$ OpenHyperInterval $(a-n \mapsto r, a+n \mapsto r)$.

ProOF: Reconsider $a=e$ as an element of $\mathcal{R}^{n}$. Reconsider $p=e$ as a point of $\mathcal{E}_{\mathrm{T}}^{n}$. Consider $e_{0}$ being a point of $\mathcal{E}^{n}$ such that $p=e_{0}$ and OpenHypercube $\left(e_{0}, r\right)=$ OpenHypercube $(p, r)$. OpenHypercube $(e, r) \subseteq$ OpenHyperInterval $(a-n \mapsto r, a+n \mapsto r)$ by [8, (27)], [6, (57)], [8, (11)], [18, (4)]. OpenHyperInterval $(a-n \mapsto r, a+n \mapsto r) \subseteq$ OpenHypercube $(e, r)$ by [10, (22)], [8, (27)], [6, (57)], [8, (11)].

(81) Let us consider a point $p$ of $\mathcal{E}_{\mathrm{T}}^{n}$. Then there exists an element $a$ of $\mathcal{R}^{n}$ such that

(i) $a=p$, and

(ii) ClosedHypercube $(p, b)=\operatorname{ClosedHyperInterval}(a-b, a+b)$.

Proof: Reconsider $a=p$ as an element of $\mathcal{R}^{n}$. ClosedHypercube $(p, b) \subseteq$ ClosedHyperInterval $(a-b, a+b)$ by [10, (22)], [8, (11), (27)]. ClosedHyperInt$\operatorname{erval}(a-b, a+b) \subseteq$ ClosedHypercube $(p, b)$ by [10, (22)], [8, (11), (27)].

\section{Correspondance Between Interval and 1-Dimensional Hyper INTERVAL}

Let us consider a real number $x$. Now we state the propositions:

(82) $x \in[r, s]$ if and only if $1 \mapsto x \in$ ClosedHyperInterval $(\langle r\rangle,\langle s\rangle)$.

Proof: Set $a_{1}=\langle r\rangle$. Set $b_{1}=\langle s\rangle$. For every real number $x$ such that $x \in[r, s]$ holds $1 \mapsto x \in$ ClosedHyperInterval $\left(a_{1}, b_{1}\right)$ by [4, (2)], [24, (7)]. For every real number $x$ such that $1 \mapsto x \in \operatorname{ClosedHyperInterval}\left(a_{1}, b_{1}\right)$ holds $x \in[r, s]$ by [24, (7)]. 
(83) $x \in] r, s[$ if and only if $1 \mapsto x \in$ OpenHyperInterval $(\langle r\rangle,\langle s\rangle)$.

Proof: Set $a_{1}=\langle r\rangle$. Set $b_{1}=\langle s\rangle$. For every real number $x$ such that $x \in] r, s\left[\right.$ holds $1 \mapsto x \in$ OpenHyperInterval $\left(a_{1}, b_{1}\right)$ by [4, (2)], 24, (7)]. For every real number $x$ such that $1 \mapsto x \in \operatorname{OpenHyperInterval}\left(a_{1}, b_{1}\right)$ holds $x \in] r, s[$ by [24, (7)].

(84) $x \in] r, s]$ if and only if $1 \mapsto x \in \operatorname{LeftOpenHyperInterval}(\langle r\rangle,\langle s\rangle)$.

Proof: Set $a_{1}=\langle r\rangle$. Set $b_{1}=\langle s\rangle$. For every real number $x$ such that $x \in] r, s]$ holds $1 \mapsto x \in \operatorname{LeftOpenHyperInterval}\left(a_{1}, b_{1}\right)$ by [4, (2)], [24, (7)]. For every real number $x$ such that $1 \mapsto x \in \operatorname{LeftOpenHyperInterval}\left(a_{1}, b_{1}\right)$ holds $x \in] r, s]$ by [24, (7)].

(85) $x \in[r, s[$ if and only if $1 \mapsto x \in \operatorname{RightOpenHyperInterval}(\langle r\rangle,\langle s\rangle)$.

Proof: Set $a_{1}=\langle r\rangle$. Set $b_{1}=\langle s\rangle$. For every real number $x$ such that $x \in$ $\left[r, s\right.$ [ holds $1 \mapsto x \in$ RightOpenHyperInterval $\left(a_{1}, b_{1}\right)$ by [4, (2)], 24, (7)]. For every real number $x$ such that $1 \mapsto x \in \operatorname{RightOpenHyperInterval}\left(a_{1}, b_{1}\right)$ holds $x \in[r, s[$ by $[24,(7)]$.

\section{Correspondance Between Measurable Rectangle and Product}

From now on $n$ denotes a non zero natural number.

Now we state the propositions:

(86) Let us consider an $n$-tuple $s$ of the set of all open real bounded intervals. Then there exist elements $a, b$ of $\mathcal{R}^{n}$ such that for every natural number $i$ such that $i \in \operatorname{Seg} n$ holds $s(i)=] a(i), b(i)[$.

Proof: $s \in$ (the set of all open real bounded intervals) $)^{\operatorname{Seg} n}$. Consider $f$ being a function such that $s=f$ and $\operatorname{dom} f=\operatorname{Seg} n$ and $\operatorname{rng} f \subseteq$ the set of all open real bounded intervals. Define $\mathcal{P}$ [object, object $] \equiv$ there exists an element $f$ of $\mathbb{R} \times \mathbb{R}$ such that $f=\$_{2}$ and $\left.s\left(\$_{1}\right)=\right](f)_{\mathbf{1}},(f)_{\mathbf{2}}[$. For every natural number $i$ such that $i \in \operatorname{Seg} n$ there exists an element $d$ of $\mathbb{R} \times \mathbb{R}$ such that $\mathcal{P}[i, d]$ by $[7,(3)]$. Consider $f$ being a finite sequence of elements of $\mathbb{R} \times \mathbb{R}$ such that len $f=n$ and for every natural number $i$ such that $i \in \operatorname{Seg} n$ holds $\mathcal{P}\left[i, f_{i}\right]$ from [25, Sch. 1]. Consider $z$ being an element of $\mathcal{R}^{n} \times \mathcal{R}^{n}$ such that for every natural number $i$ such that $i \in \operatorname{Seg} n$ holds $(z)_{\mathbf{1}}(i)=\left(f_{i}\right)_{\mathbf{1}}$ and $(z)_{\mathbf{2}}(i)=\left(f_{i}\right)_{\mathbf{2}}$. Reconsider $a=(z)_{\mathbf{1}}, b=(z)_{\mathbf{2}}$ as an element of $\mathcal{R}^{n}$. For every natural number $i$ such that $i \in \operatorname{Seg} n$ holds $s(i)=] a(i), b(i)[$.

(87) Let us consider an element $x$ of $\operatorname{Product}(n$, the set of all open real bounded intervals). Then there exist elements $a, b$ of $\mathcal{R}^{n}$ such that for every element $t$ of $\mathcal{R}^{n}, t \in x$ iff for every natural number $i$ such that 
$i \in \operatorname{Seg} n$ holds $t(i) \in] a(i), b(i)[$. The theorem is a consequence of $(62)$ and (86).

(88) Let us consider an $n$-tuple $s$ of the set of all left open real bounded intervals. Then there exist elements $a, b$ of $\mathcal{R}^{n}$ such that for every natural number $i$ such that $i \in \operatorname{Seg} n$ holds $s(i)=] a(i), b(i)]$.

Proof: $s \in$ (the set of all left open real bounded intervals) ${ }^{\operatorname{Seg} n}$. Consider $f$ being a function such that $s=f$ and $\operatorname{dom} f=\operatorname{Seg} n$ and $\operatorname{rng} f \subseteq$ the set of all left open real bounded intervals. Define $\mathcal{P}$ [object, object] $\equiv$ there exists an element $f$ of $\mathbb{R} \times \mathbb{R}$ such that $f=\$_{2}$ and $\left.\left.s\left(\$_{1}\right)=\right](f)_{\mathbf{1}},(f)_{\mathbf{2}}\right]$. For every natural number $i$ such that $i \in \operatorname{Seg} n$ there exists an element $d$ of $\mathbb{R} \times \mathbb{R}$ such that $\mathcal{P}[i, d]$ by $[7,(3)]$. Consider $f$ being a finite sequence of elements of $\mathbb{R} \times \mathbb{R}$ such that len $f=n$ and for every natural number $i$ such that $i \in \operatorname{Seg} n$ holds $\mathcal{P}\left[i, f_{i}\right]$ from [25, Sch. 1]. Consider $z$ being an element of $\mathcal{R}^{n} \times \mathcal{R}^{n}$ such that for every natural number $i$ such that $i \in \operatorname{Seg} n$ holds $(z)_{\mathbf{1}}(i)=\left(f_{i}\right)_{\mathbf{1}}$ and $(z)_{\mathbf{2}}(i)=\left(f_{i}\right)_{\mathbf{2}}$. Reconsider $a=(z)_{\mathbf{1}}, b=(z)_{\mathbf{2}}$ as an element of $\mathcal{R}^{n}$. For every natural number $i$ such that $i \in \operatorname{Seg} n$ holds $s(i)=] a(i), b(i)]$.

(89) Let us consider an element $x$ of $\operatorname{Product}(n$, the set of all left open real bounded intervals). Then there exist elements $a, b$ of $\mathcal{R}^{n}$ such that for every element $t$ of $\mathcal{R}^{n}, t \in x$ iff for every natural number $i$ such that $i \in \operatorname{Seg} n$ holds $t(i) \in] a(i), b(i)]$. The theorem is a consequence of (62) and (88).

(90) Let us consider an $n$-tuple $s$ of the set of all right open real bounded intervals. Then there exist elements $a, b$ of $\mathcal{R}^{n}$ such that for every natural number $i$ such that $i \in \operatorname{Seg} n$ holds $s(i)=[a(i), b(i)[$.

Proof: $s \in$ (the set of all right open real bounded intervals) ${ }^{\operatorname{Seg} n}$. Consider $f$ being a function such that $s=f$ and $\operatorname{dom} f=\operatorname{Seg} n$ and $\operatorname{rng} f \subseteq$ the set of all right open real bounded intervals. Define $\mathcal{P}$ [object, object] $\equiv$ there exists an element $f$ of $\mathbb{R} \times \mathbb{R}$ such that $f=\$_{2}$ and $s\left(\$_{1}\right)=\left[(f)_{\mathbf{1}},(f)_{\mathbf{2}}[\right.$. For every natural number $i$ such that $i \in \operatorname{Seg} n$ there exists an element $d$ of $\mathbb{R} \times \mathbb{R}$ such that $\mathcal{P}[i, d]$ by [7, (3)]. Consider $f$ being a finite sequence of elements of $\mathbb{R} \times \mathbb{R}$ such that len $f=n$ and for every natural number $i$ such that $i \in \operatorname{Seg} n$ holds $\mathcal{P}\left[i, f_{i}\right]$ from [25, Sch. 1]. Consider $z$ being an element of $\mathcal{R}^{n} \times \mathcal{R}^{n}$ such that for every natural number $i$ such that $i \in \operatorname{Seg} n$ holds $(z)_{\mathbf{1}}(i)=\left(f_{i}\right)_{\mathbf{1}}$ and $(z)_{\mathbf{2}}(i)=\left(f_{i}\right)_{\mathbf{2}}$. Reconsider $a=(z)_{\mathbf{1}}, b=(z)_{\mathbf{2}}$ as an element of $\mathcal{R}^{n}$. For every natural number $i$ such that $i \in \operatorname{Seg} n$ holds $s(i)=[a(i), b(i)[$.

(91) Let us consider an element $x$ of $\operatorname{Product}(n$, the set of all right open real bounded intervals). Then there exist elements $a, b$ of $\mathcal{R}^{n}$ such that 
for every element $t$ of $\mathcal{R}^{n}, t \in x$ iff for every natural number $i$ such that $i \in \operatorname{Seg} n$ holds $t(i) \in[a(i), b(i)[$. The theorem is a consequence of (62) and (90).

(92) MeasurableRectangle LeftOpenIntervals $(n)=\operatorname{Product}(n$, the set of all left open real bounded intervals). The theorem is a consequence of (40) and (66).

(93) MeasurableRectangle RightOpenIntervals $(n)=\operatorname{Product}(n$, the set of all right open real bounded intervals). The theorem is a consequence of (46) and (67).

\section{Chebyshev Distance}

In the sequel $n$ denotes a non zero natural number and $x, y, z$ denote elements of $\mathcal{R}^{n}$.

Let us consider $n$. The functor $D_{\text {Chebyshev }}^{n}$ yielding a function from $\mathcal{R}^{n} \times \mathcal{R}^{n}$ into $\mathbb{R}$ is defined by

(Def. 20) for every elements $x, y$ of $\mathcal{R}^{n}$, it $(x, y)=\sup \operatorname{rng}|x-y|$.

Now we state the propositions:

(94) (i) the set of all $|x(i)-y(i)|$ where $i$ is an element of $\operatorname{Seg} n$ is realmembered, and

(ii) the set of all $|x(i)-y(i)|$ where $i$ is an element of $\operatorname{Seg} n=\operatorname{rng}|x-y|$. Proof: Set $S=$ the set of all $|x(i)-y(i)|$ where $i$ is an element of $\operatorname{Seg} n$. $S \subseteq \operatorname{rng}|x-y|$ by [8, (27)], [6, (124)]. For every object $t$ such that $t \in$ $\operatorname{rng}|x-y|$ holds $t \in S$ by [6, (124)], [8, (27)].

(95) There exists an extended real-membered set $S$ such that

(i) $S=$ the set of all $|x(i)-y(i)|$ where $i$ is an element of $\operatorname{Seg} n$, and

(ii) $\left(D_{\text {Chebyshev }}^{n}\right)(x, y)=\sup S$.

The theorem is a consequence of (94).

(96) $\left(D_{\text {Chebyshev }}^{n}\right)(x, y)=|x-y|(\max -\operatorname{diff}-\operatorname{index}(x, y))$.

ProOf: $\left(D_{\text {Chebyshev }}^{n}\right)(x, y) \leqslant|x-y|(\max -\operatorname{diff}-\operatorname{index}(x, y))$ by [15, (5)].

(97) $\left(D_{\text {Chebyshev }}^{n}\right)(x, y)=0$ if and only if $x=y$.

Proof: Consider $S$ being an extended real-membered set such that $S=$ the set of all $|x(i)-y(i)|$ where $i$ is an element of $\operatorname{Seg} n$ and $\left(D_{\text {Chebyshev }}^{n}\right)(x, y)=\sup S . S=\{0\}$ by [19, (2)], [3, (53)], [4, (1)].

(98) $\left(D_{\text {Chebyshev }}^{n}\right)(x, y)=\left(D_{\text {Chebyshev }}^{n}\right)(y, x)$. The theorem is a consequence of (1). 
(99) $\left(D_{\text {Chebyshev }}^{n}\right)(x, y) \leqslant\left(D_{\text {Chebyshev }}^{n}\right)(x, z)+\left(D_{\text {Chebyshev }}^{n}\right)(z, y)$.

PROOF: Reconsider $s_{1}=\sup \operatorname{rng}|x-y|, s_{2}=\sup \operatorname{rng}|x-z|, s_{3}=\sup \operatorname{rng} \mid z-$ $y \mid$ as a real number. $s_{1} \leqslant s_{2}+s_{3}$ by [8, (27)], [5, (56)], [6, (124)], (2).

(100) $D_{\text {Chebyshev }}^{n}$ is a metric of $\mathcal{R}^{n}$. The theorem is a consequence of (97), (98), and (99).

(101) $\rho^{2}([0,0],[1,1])=\sqrt{2}$.

(102) $\left(D_{\text {Chebyshev }}^{2}\right)([0,0],[1,1])=1$.

Proof: Consider $S$ being an extended real-membered set such that $S=$ the set of all $|[0,0](i)-[1,1](i)|$ where $i$ is an element of Seg 2 and $\left(D_{\text {Chebyshev }}^{2}\right)([0,0],[1,1])=\sup S . S=\{|0-1|\}$ by [4, (2), (44)].

Let us consider elements $x, y$ of $\mathcal{R}^{1}$. Now we state the propositions:

(103) $\left(D_{\text {Chebyshev }}^{1}\right)(x, y)=|x(1)-y(1)|$.

Proof: Consider $S$ being an extended real-membered set such that $S=$ the set of all $|x(i)-y(i)|$ where $i$ is an element of Seg 1 and

$\left(D_{\text {Chebyshev }}^{1}\right)(x, y)=\sup S . S=\{|x(1)-y(1)|\}$ by [4, (2)].

(104) $\rho^{1}(x, y)=|x(1)-y(1)|$.

Now we state the propositions:

(105) $\rho^{1}=D_{\text {Chebyshev }}^{1}$. The theorem is a consequence of (104) and (103).

(106) $\rho^{2} \neq D_{\text {Chebyshev }}^{2}$. The theorem is a consequence of (101) and (102).

Let $n$ be a non zero natural number. The functor $\mathrm{L}_{\infty}(n)$ yielding a strict metric space is defined by the term

(Def. 21) $\left\langle\mathcal{R}^{n}, D_{\text {Chebyshev }}^{n}\right\rangle$.

Let us observe that $\mathrm{L}_{\infty}(n)$ is non empty.

The functor $\mathcal{E}_{\infty}^{n}(n)$ yielding a strict real linear topological structure is defined by

(Def. 22) the topological structure of it $=\left(\mathrm{L}_{\infty}(n)\right)_{\text {top }}$ and the RLS structure of $i t=\mathbb{R}_{\mathbb{R}}^{\operatorname{Seg} n}$.

Now we state the proposition:

(107) The RLS structure of $\mathcal{E}_{\mathrm{T}}^{n}=$ the RLS structure of $\mathcal{E}_{\infty}^{n}(n)$.

Let $n$ be a non zero natural number. Let us note that $\mathcal{E}_{\infty}^{n}(n)$ is non empty.

Now we state the propositions:

(108) Let us consider an element $x$ of $\mathcal{R}^{0}$. Then

(i) $\operatorname{Intervals}(x, r)$ is empty, and

(ii) $\prod \operatorname{Intervals}(x, r)=\{\emptyset\}$.

(109) If $r \leqslant 0$, then $\prod \operatorname{Intervals}(x, r)$ is empty. 
In the sequel $p$ denotes an element of $\mathrm{L}_{\infty}(n)$.

Let $n$ be a non zero natural number and $p$ be an element of $\mathrm{L}_{\infty}(n)$. The functor ${ }^{\circledR} p$ yielding an element of $\mathcal{R}^{n}$ is defined by the term

(Def. 23) $p$.

Now we state the propositions:

(110) $\operatorname{Ball}(p, r)=\prod \operatorname{Intervals}\left({ }^{\circledR} p, r\right)$. The theorem is a consequence of (109), (95), and (96).

(111) Let us consider a point $e$ of $\mathcal{E}^{n}$. If $e=p$, then $\operatorname{Ball}(p, r)=$ OpenHypercube $(e, r)$. The theorem is a consequence of (110).

Let $n$ be a non zero natural number, $p$ be an element of $\mathrm{L}_{\infty}(n)$, and $r$ be a negative real number. Let us note that $\overline{\operatorname{Ball}}(p, r)$ is empty.

Now we state the propositions:

(112) Let us consider an object $t$. Then $t \in \overline{\operatorname{Ball}}(p, r)$ if and only if there exists a function $f$ such that $t=f$ and $\operatorname{dom} f=\operatorname{Seg} n$ and for every natural number $i$ such that $i \in \operatorname{Seg} n$ holds $f(i) \in\left[\left({ }^{@} p\right)(i)-r,\left({ }^{@} p\right)(i)+r\right]$. The theorem is a consequence of (95).

(113) Let us consider a point $p$ of $\mathcal{E}_{\mathrm{T}}^{n}$, and an element $q$ of $\mathrm{L}_{\infty}(n)$. Suppose $q=p$. Then $\overline{\operatorname{Ball}}(q, r)=$ ClosedHypercube $(p, n \mapsto r)$.

PROOF: For every object $x$ such that $x \in \overline{\operatorname{Ball}}(q, r)$ holds $x \in$ ClosedHypercube $(p, n \mapsto r)$ by (112), [6, (57), (93)], [10, (22)]. For every object $x$ such that $x \in \operatorname{ClosedHypercube}(p, n \mapsto r)$ holds $x \in \overline{\operatorname{Ball}}(q, r)$ by [10, (22)], [6, (131), (124), (57)].

(114) $\operatorname{Ball}(p, r)=$ OpenHyperInterval $\left({ }^{@} p-n \mapsto r,{ }^{\circledR} p+n \mapsto r\right)$. The theorem is a consequence of (80) and (110).

(115) $\overline{\operatorname{Ball}}(p, r)=$ ClosedHyperInterval $\left({ }^{\circledR} p-n \mapsto r,{ }^{\circledR} p+n \mapsto r\right)$. The theorem is a consequence of (81) and (113).

\section{REFERENCES}

[1] Grzegorz Bancerek. König's theorem. Formalized Mathematics, 1(3):589-593, 1990.

[2] Grzegorz Bancerek. On powers of cardinals. Formalized Mathematics, 3(1):89-93, 1992.

[3] Grzegorz Bancerek. The fundamental properties of natural numbers Formalized Mathematics, 1(1):41-46, 1990.

[4] Grzegorz Bancerek and Krzysztof Hryniewiecki. Segments of natural numbers and finite sequences. Formalized Mathematics, 1(1):107-114, 1990.

[5] Czesław Byliński. The complex numbers. Formalized Mathematics, 1(3):507-513, 1990.

[6] Czesław Byliński. Finite sequences and tuples of elements of a non-empty sets. Formalized Mathematics, 1(3):529-536, 1990.

[7] Czesław Byliński. Functions and their basic properties. Formalized Mathematics, 1(1): 55-65, 1990.

[8] Czesław Byliński. The sum and product of finite sequences of real numbers Formalized Mathematics, 1(4):661-668, 1990. 
[9] Czesław Byliński. Some basic properties of sets Formalized Mathematics, 1(1):47-53, 1990.

[10] Agata Darmochwał. The Euclidean space. Formalized Mathematics, 2(4):599-603, 1991.

[11] Michel Marie Deza and Elena Deza. Encyclopedia of distances. Springer, 2009.

[12] Noboru Endou, Katsumi Wasaki, and Yasunari Shidama. Definitions and basic properties of measurable functions Formalized Mathematics, 9(3):495-500, 2001.

[13] Adam Grabowski. On the subcontinua of a real line Formalized Mathematics, 11(3): 313-322, 2003.

[14] Adam Grabowski. On the Borel families of subsets of topological spaces. Formalized Mathematics, 13(4):453-461, 2005.

[15] Artur Korniłowicz. The correspondence between $n$-dimensional Euclidean space and the product of $n$ real lines. Formalized Mathematics, 18(1):81-85, 2010. doi:10.2478/v10037010-0011-0.

[16] Jean Mawhin. Analyse: fondements, techniques, évolution. De Boeck, 1992.

[17] Beata Padlewska. Families of sets Formalized Mathematics, 1(1):147-152, 1990.

[18] Karol Pąk. Tietze extension theorem for $n$-dimensional spaces. Formalized Mathematics, 22(1):11-19, 2014. doi 10.2478/forma-2014-0002.

[19] Jan Popiołek. Some properties of functions modul and signum. Formalized Mathematics, $1(2): 263-264,1990$.

[20] Marco Riccardi. The definition of topological manifolds. Formalized Mathematics, 19(1): 41-44, 2011. doi $10.2478 / \mathrm{v} 10037-011-0007-4$.

[21] Marco Riccardi. Planes and spheres as topological manifolds. Stereographic projection. Formalized Mathematics, 20(1):41-45, 2012. doi 10.2478/v10037-012-0006-0

[22] Jean Schmets. Analyse mathematique Notes de cours, Université de Liège, 337 pages, 2004.

[23] Alexander Yu. Shibakov and Andrzej Trybulec. The Cantor set Formalized Mathematics, 5(2):233-236, 1996.

[24] Andrzej Trybulec. Binary operations applied to functions Formalized Mathematics, 1 (2):329-334, 1990.

[25] Wojciech A. Trybulec. Pigeon hole principle Formalized Mathematics, 1(3):575-579, 1990.

[26] Wojciech A. Trybulec. Basis of real linear space. Formalized Mathematics, 1(5):847-850, 1990.

[27] Edmund Woronowicz. Relations defined on sets. Formalized Mathematics, 1(1):181-186, 1990. 